\title{
Quaderni
}

QUADERNI Communication, technologies, pouvoir

67| Automne 2008

Jeu vidéo et discours

\section{Le droit contre les usages : la crémation, destination et statut des cendres}

\section{Gaëlle Clavandier}

\section{(2) OpenEdition}

1 Journals

\section{Édition électronique}

URL : http://journals.openedition.org/quaderni/238

DOI : 10.4000/quaderni.238

ISSN : 2105-2956

\section{Éditeur}

Les éditions de la Maison des sciences de l'Homme

\section{Édition imprimée}

Date de publication : 5 octobre 2008

Pagination : 107-124

\section{Référence électronique}

Gaëlle Clavandier, «Le droit contre les usages : la crémation, destination et statut des cendres », Quaderni [En ligne], 67 | Automne 2008, mis en ligne le 05 janvier 2012, consulté le 19 avril 2019. URL http://journals.openedition.org/quaderni/238; DOI : 10.4000/quaderni.238 


\section{Politique}

\section{le droit contre} les usages : la crémation, destination et statut des cendres

\section{Gaëlle Clavandier}

Maître de conférences en sociologie Université Lyon MODYS, CNRS
La crémation en France sort actuellement de son statut de pratique marginale, au sens de pratique relativement peu répandue et peu normative, pour devenir un référentiel en matière de choix de sépultures au même titre que peut l'être l'inhumation'. Il va de soi que cette question des modes de sépulture devient cruciale en raison de l'évolution statistique de la pratique crématiste. À partir du moment où l'usage est massif (en 2005, environ 110000 crémations ont été pratiquées) et lorsque les vœux affichés d'avoir recours à cette technique se multiplient, il n'est plus possible de percevoir cette démarche comme une intention atypique ${ }^{2}$. La tombe demeure un idéal-type, cependant elle ne constituerait plus le seul modèle opérant. Ce changement de mœurs implique plus largement que le simple fait de recourir à un mode de sépulture. C'est toute la culture du culte du tombeau ${ }^{3}$, du souvenir situé des morts qui se trouve questionné. Or, fruit des siècles passés, le bouleversement qui nous est donné à voir est d'autant plus frappant que l'ouverture ainsi produite induirait des réactions en chaîne, une complète redéfinition du rapport à la mort. Certes, la crémation est une pratique ancestrale, mais pas telle qu'elle s'actualise en France ${ }^{4}$. S'il est notable que depuis deux décennies au moins la crémation ne pose plus de problème, il en va autrement de l'usage des cendres qui en découlent. La ligne de partage n'est plus de promouvoir l'inhumation au détriment de la crémation vue comme une «manifestation d'irréligion» par le clergés, mais de penser la crémation sur un autre plan que celui de l'inhumation, laquelle suppose une « sédentarisation» des morts hors champ des vivants. Or, la réponse actuelle des pouvoirs publics va davantage dans le sens d'une 
harmonisation des pratiques autour d'un unique modèle se référant à une tradition, que dans le sens de l'invention d'un nouveau rituel.

L'intention de cet article est d'analyser les transformations en cours au regard du droit. En effet, si la crémation offre un cadre actuellement plus souple que l'inhumation avec la possibilité de conserver l'urne cinéraire à domicile, de disperser les cendres en tout lieu autre que la voie publique ou de déposer l'urne dans un caveau familial, un cavurne ou un columbarium, celuici est remis en cause. La proposition de loi du 22 juin 2006 adoptée en première lecture au Sénat vise : à mieux encadrer la crémation mais aussi à contrôler la qualification des opérateurs funéraires, à améliorer la formation professionnelle des personnels et à simplifier les démarches des familles ${ }^{6}$. Le débat étant plus vif au sujet de la crémation, notamment sur la destination ultime des cendres, leur utilisation, leur statut, nous nous proposons de porter notre attention sur cet axe en particulier. Outre l'analyse des pratiques et des représentations contemporaines de la crémation, notre propos se fonde sur l'étude de la proposition de loi ainsi que sur le compte rendu intégral des débats au Sénat, soit un texte d'une cinquantaine de pages.

En quoi une société a-t-elle nécessité de recourir à un encadrement légal à propos de la crémation ? Pourquoi semble-t-il obligatoire de doter les cendres d'un statut légal alors que tel n'avait pas été le cas pour le cadavre ? Comment justifier le fait qu'il ne sera plus possible de conserver les cendres au domicile des proches du défunt? Pourquoi valider de façon légale un culte du souvenir avec au centre le cimetière, alors même que la crémation d'un point de vue pratique et philosophique reconnaît la possibilité d'une dissipation des cendres ? Autant de questions que le sociologue doit se poser afin de mesurer et comprendre les mutations en cours. Il s'agit de voir comment le droit s'invite dans la construction d'une norme sociale, alors que des comportements hétéroclites se multiplient peinant à dire ce qui fait le propre de la crémation aujourd'hui en France. Entre publicisation et privatisation, entre normalisation et liberté individuelle, le travail de la loi est l'objet d'une tension entre ce qui se doit d'être encadré et ce qui relève des choix intimes des individus, d'où cette posture qui vise à « encadrer la liberté $»^{7}$. Or, en la matière les consensus se négocient âprement et c'est le registre de la morale qui bien souvent fait tampon. Comment assurer la libre disposition des cendres tout en encadrant leur destination ? Comment trouver un équilibre entre le principe du respect dû aux défunts et le principe de liberté des funérailles ? C'est le défi que s'est fixé le législateur. Gageure, pari audacieux, engagement éthique c'est selon, une chose est sûre, l'entremise du droit fait œuvre de normalisation sans réellement lever les ambiguïtés.

\section{La proposition de loi du 22 juin 2006}

Avant tout chose, il est indispensable de présenter la spécificité du cadre juridique tel qu'il devrait advenir. La proposition de loi votée au Sénat émane de J.-P. Sueur, sénateur du Loiret, qui a eu l'initiative de plusieurs lois et décrets sur le sujet ${ }^{8}$. Elle est basée sur un rapport de J.-R. Lecerf commandité par la commission des lois constitutionnelles, de la législation et de l'administration générale de la République. 
Composée de cinq chapitres, elle a trait :

- au renforcement des conditions d'exercice de la profession d'opérateur funéraire ;

- à la simplification et la sécurisation des démarches des familles;

- au statut et à la destination des cendres des personnes décédées dont le corps a donné lieu à crémation ;

- à la conception et à la gestion des cimetières ;

- aux dispositions diverses et transitoires.

C'est le chapitre trois de la loi, le plus volumineux mais aussi le plus problématique qui va nous intéresser ici.

Les articles 16-1 et 16-2 du Code civil sont modifiés. Désormais, le respect dû au corps humain ne cesse pas avec la mort : «Les restes des personnes décédées, y compris les cendres de celles dont le corps a donné lieu à crémation, doivent être traités avec respect, dignité et décence " (Article 9 de la proposition de loi n³186, du 22 juin 2006). Dans les articles pré-existants, il est précisé que toutes les sépultures, dont les urnes cinéraires, répondent aux mêmes obligations.

Les articles L. 2223-1 et L. 2223-2 du Code général des collectivités territoriales prennent en compte le changement que suppose la crémation en termes de gestion de l'espace funéraire. Ainsi, la loi prévoit que les communes de plus de 10000 habitants devront se doter d'un site cinéraire destiné à l'accueil des cendres. Qui plus est, «le site cinéraire (...) comprend un espace aménagé pour leur dispersion et doté d'un équipement mentionnant l'identité des défunts, ainsi qu'un columbarium ou des caveaux d'urnes appelés cavurnes » (Article 13 de la proposition de loi n³186, du 22 juin 2006).

Une sous-section du Code des collectivités territoriales est désormais consacrée à la destination des cendres, mentionnant de fait la place accordée par le législateur à cette question. Les articles L. 2223-18-1, L. 2223-18-2, L. 2223-183 et L. 2223-18-4 visent à sécuriser et encadrer les pratiques relatives au devenir des cendres. Il est stipulé que les cendres sont pulvérisées et recueillies immédiatement dans une urne cinéraire, laquelle est munie extérieurement d'une plaque portant l'identité du défunt et le nom du crématorium. L'urne, en l'attente d'une décision quant à sa destination, peut-être conservée au crématorium durant une période qui ne peut excéder six mois. Au terme de ce délai, si aucune démarche n'a été faite par les proches, les cendres sont dispersées dans l'espace aménagé à cet effet, soit l'espace communément nommé jardin $d u$ souvenir. Trois possibilités sont désormais octroyées aux familles (du point de vue légal il s'agit de « la personne ayant qualité pour pourvoir aux funérailles ») :

- la conservation de l'urne cinéraire pouvant être déposée dans une sépulture, une case de columbarium ou un cavurne, ou scellée sur un monument funéraire à l'intérieur d'un cimetière ou d'un site cinéraire ;

- la dispersion dans un espace aménagé à cet effet d'un cimetière ou un site cinéraire ;

- la dispersion en pleine nature, sauf sur les voies publiques.

Dorénavant, la conservation d'une urne à titre privé sera impossible (c'est du moins le projet de 
la proposition de loi) et la dispersion des cendres s'accompagne d'une déclaration à la mairie de la commune du lieu du décès; l'identité du défunt, la date et le lieu de la dispersion devant être consignés sur un registre créé à cet effet ${ }^{9}$. Toute personne à titre gratuit ou onéreux qui utiliserait ou gérerait un lieu collectif en dehors du cimetière public destiné au dépôt temporaire ou définitif d'urnes ou de cendres se verrait puni d'une amende de 15000 euros.

L'article L. 2223-40 précise que ce sont les communes et les établissements publics de coopération intercommunale qui sont seuls compétents pour créer et gérer les crématoriums et les sites cinéraires. Toute création de crématorium ou extension donne lieu à un avis préfectoral. La gestion des crématoriums devient une nécessité que la loi entérine. Chaque région se dote d'un «schéma régional des crématoriums» qui vise : à recenser les équipements existants, à produire une évaluation prospective et à faire mention des équipements à créer. Les établissements privés sont de ce fait interdits.

Le chapitre quatre de la proposition de loi, signale que si aucune mention n'a été explicitement faite, tout corps dont la concession est close peut donner lieu à crémation. En conséquence, la loi prévoit le cas où, pour des raisons confessionnelles notamment, une personne souhaite que son corps ne soit jamais réduit en cendres. Dès lors, «les restes de personnes qui avaient manifesté leur opposition à la crémation sont distingués au sein de l'ossuaire " (Article 18 de la proposition de loi $\mathrm{n}^{\circ} 3186$, du 22 juin 2006).
Présentée ainsi, dans sa structure même, la loi paraît simple, peu polémique. Pourtant, si tout le monde ou presque s'accorde sur la nécessité d'une prise en compte de la spécificité de la crémation, cette spécificité est en partie gommée, puisque le texte de loi associe le plus souvent crémation et inhumation, cendres et corps. Cette ambivalence de la loi peut expliquer en partie le fait que le débat demeure très vif sur certains aspects. Statuer sur le devenir des cendres, plus généralement sur les pratiques funéraires, c'est s'interroger sur un choix de société. La place des morts ${ }^{10}$ occupe un espace bien plus important que le simple cimetière, dont la mise en demeure en périphérie de la ville ne doit pas occulter que les morts sont là parmi les vivants, outre le souvenir, il va de soi. La dynamique des cendres, lesquelles sont fluides, volatiles, éphémères, quasi-invisibles, présuppose une occupation du territoire bien plus élargie que pour le cadavre. Ainsi réduite, la matière du corps oblige à spécifier la réponse alors même que la loi doit être identique pour tous, par-delà la mort.

Cette proposition de loi, si elle répond à de longs questionnements et discussions, paraît désormais primordiale aux yeux du législateur, d'où sa mise sur agenda. Au terme d'une quarantaine d'auditions, la commission formulait vingtsept recommandations "destinées à assurer la sérénité des vivants et le respect des défunts » (J.-R. Lecerf, discussion en séance publique au Sénat le 22 juin 2006). Ces recommandations s'articulent autour d'une nécessaire gestion des funérailles et d'un indispensable recours aux valeurs. En appeler à une organisation des funérailles et du souvenir reposant sur un cadre sécurisant suppose de repenser dans son 
ensemble la législation funéraire. Plutôt que de promulguer au coup par coup des décrets ou d'ordonner des arrêtés, le bien-fondé d'une telle loi se base sur le constat d'un changement rapide des pratiques, donc sur la nécessité de les doter d'un cadre - lequel est supposé défaillant tant les comportements actuels semblent épars et parfois inadaptés. Il est ainsi prévu de mieux encadrer les opérateurs funéraires qui ont acquis une réelle autonomie depuis la loi de 1993. Il s'agit également de protéger les familles endeuillées supposées affaiblies lors d'un deuil et dans l'incapacité matérielle et psychologique de se prémunir contre les abus (d'où une demande de transparence des prix avec l'établissement de devis). Il est question d'apporter une réponse au développement des crémations tant du point de vue des équipements et des services que de la place faite à ces défunts crématisés. Or, la législation en vigueur est très souple en matière de crémation, donnant lieu à une grande diversité de comportements. En couplant la morale et le droit, l'idée est de contrôler les pratiques répréhensibles. Mais au-delà de ce cadrage, le législateur n'hésite pas à dire qu'il énonce un projet de société.

Le fondement d'une telle approche n'est pas questionné et il est étayé par des contraintes tangibles : l'impératif d'agir dans un contexte en transformation; le devoir de condamner des comportements déviants et par conséquent d'édifier les bonnes pratiques. Il est intéressant de constater que toute pratique qui sortirait de près ou de loin d'un cadre moral devient de fait déviante. Comme la loi jusque-là énonce peu de prescriptions, l'inconnu, à savoir tout ce qui ne ressemblerait pas à un usage apaisé des cendres, est proscrit. L'imaginaire de l'immobilité (fixation des morts en un lieu et une temporalité) se décline-t-il en une demande d'immobilisme qui irait jusqu'à une sédentarisation exclusive des cendres dans des sites cinéraires ? L'observateur perçoit d'emblée dans le texte de loi deux registres de justification qui se superposent, l'un moral, l'autre politique : avec la mise en exergue d'une moralisation des pratiques en promouvant la décence, le respect et la dignité et plus généralement la personne dans son intimité ; avec la convocation de valeurs laïques et républicaines prônant l'égalité des modes de sépultures. De fait, la loi propose de penser et d'outiller la crémation dans un espace culturel déjà défini, celui d'un espace public renforçant le culte du souvenir.

En conséquence de quoi le droit veut établir une nouvelle norme ou plus précisément un nouveau registre de normativité et s'auto-promeut garant d'un équilibre futur. Si la spécificité de l'herméneutique juridique est de supprimer la subjectivité de l'interprète, elle se caractérise par l'objectivité et la rationalité en ayant une fonction avant tout pragmatique. Si la nécessité de supprimer l'aléa dans les décisions qui relèvent du droit font que l'interprétation doit être limitée à un cadre strict et technique ${ }^{11}$. Et bien, dans des cas tels que ceux-ci, la loi qui n'est certes pas le droit, se dote d'un registre de prescriptions qui relève parfois davantage de la philosophie que de la fonctionnalité pratique, l'esprit singulier de la loi offrant des dispositions plurielles.

\section{Promouvoir légalement le respect des morts}

À ne point s'y tromper, les promoteurs marquent 
la mesure d'une telle loi. Il n'est pas question d'amender, de préciser un cadre juridique déjà présent, mais bien de procéder à l'invention de ce cadre. Un réel consensus ressort de l'examen de la proposition de loi. Malgré des dissensions, l'ensemble des sénateurs est d'accord pour dire l'enjeu de ce texte et chacun s'en félicite. L'on évoque une "cause commune » inhabituelle dans l'hémicycle, et la «belle unanimité » que ce texte recueille (débat inaugural au Sénat, discussion en séance publique, le 22 juin 2006). Très peu d'amendements ont été déposés. La nécessité d'une loi prévaut donc sur tous les autres aspects.

L'exigence de doter le cadavre d'un statut et plus précisément le « cadavre d'un corps qui a donné lieu à crémation », capte le consentement de tous. À la différence des sépultures classiques, les cendres recueillies dans l'urne cinéraire n'ont pas jusqu'à lors de véritable statut. Les cendres ne sont pas assimilées par le droit à des restes humains, pas plus que le cadavre d'ailleurs. En droit, la notion de personne repose sur la protection $\mathrm{du}$ corps humain vivant, par conséquent le cadavre n'est pas une personne du point de vue juridique, mais pas tout à fait une chose non plus comme en atteste la jurisprudence. Ainsi, la prégnance de la tradition religieuse est telle que le droit, par définition laïc, en est empreint. "Par-là, le droit sanctionne l'attachement que les contemporains continuent de porter au cadavre dans lequel ils reconnaissent la dernière incarnation d'une personne qui n'est plus. En cela, le droit se fait l'écho d'une superstition de la chair ${ }^{12}$. Dès lors, si le cadavre n'a pas lui-même de véritable statut juridique, la jurisprudence l'assimile à une relique de la personne humaine faisant de lui un être, une personne à protéger. À un autre niveau, J.-D. Urbain remarque la place accordée au corps dans les pratiques funéraires. Il montre que la société contemporaine valorise à outrance le corps pour mieux évacuer la réalité affligeante du cadavre et le processus de thanatomorphose qui suit le décès ${ }^{13}$.

Le rapport préalable de la commission des lois constitutionnelles sur lequel se fonde la proposition de loi, insiste sur les abus, sur les actes dits déviants, qui, répertoriés, sont présentés en contre-exemples. La morale doit être le garant de toute pratique qui touche au domaine funéraire. Le triple credo « respect, dignité et décence» mentionné par la loi, est détaillé avec minutie. Il vient mettre à mal des pratiques jugées indécentes. Le socle de la loi, s'il s'appuie sur des cas concrets - lesquels ont été repris par les médias, puis sujets de conversation pour tous, repose tout de même sur de grands principes philosophiques et moraux. Au-delà du « cas », c'est le rapport à la mort et au corps sans vie qui est envisagé ici. L'exemple de la transformation des cendres en diamant est devenu un fait médiatique et a cristallisé les craintes de débordements ${ }^{14}$. De même, la possibilité d'envoyer des cendres en orbite spatiale est problématique ${ }^{15}$. Pourtant ce sont les pratiques quotidiennes qui dérangent davantage. Peu visibles, elles heurtent les consciences dès lors qu'elles sont identifiées. Des cendres déversées dans une décharge publique, des familles qui se disputent les restes d'un défunt, une urne déposée aux objets trouvés ou vendue dans une brocante, des œuvres d'art ou objets utilisant des cendres de personnes crématisées, autant de cas qui produisent une juxtaposition de sens entre l'être et la chose, entre le déchet et les restes humains ${ }^{16}$. Or, 
la philosophie crématiste peut pousser la logique de la réification des cendres à son paroxysme en les assimilant à une chose inerte, télescopant de la sorte les valeurs fondamentales relatives au corps. Au sujet de la division des cendres, le droit a statué à plusieurs reprises. La cour d'appel de Paris a accepté la demande conjointe d'une épouse et d'une fille de disposer des cendres de leur mari et père conformément aux dernières volontés du défunt ${ }^{17}$. Par contre, le respect des textes exclu le vœu de cette concubine de se voir attribuer l'urne et privilégie la famille, la première ne pouvant se prévaloir « d'un lien familial ». Selon la Cour d'appel de Douai ${ }^{18}$, dans ce cas les cendres n'ont pas à être partagées car l'article R. 2213-39 du Code général des collectivités territoriales précise que l'urne est « remise à la personne qui a qualité pour pourvoir aux funérailles ».

L'environnement légal dont il est désormais question prend la forme de l'offre d'un cadre juridique sécurisé, c'est du moins ce sur quoi insiste le législateur. L'absence de réel statut des cendres résultant d'une incinération provoque un débat sur l'être et la chose. Avec l'inhumation, le cadavre est pris en charge dans un avant et après l'enterrement ${ }^{19}$. Alors que pour la crémation, si de nombreuses règles viennent organiser la phase transitoire de la transformation du corps au crématorium ${ }^{20}$, peu de préconisations existent concernant la conduite à tenir à l'égard des cendres. Légiférer sur le statut des cendres des personnes décédées dont le corps a donné lieu à crémation devenait une nécessité, une nécessité reconnue par l'ensemble des sénateurs. Certaines pratiques revenaient à objectiver les cendres, lesquelles perdaient du même coup leur statut de restes humains ${ }^{21}$. Cette situation est jugée contraire à l'esprit du culte du souvenir.

L'importance accordée au statut des cendres provoque une juxtaposition de sens assez peu lisible mais emblématique dans l'esprit du législateur. Insister sur le respect, la dignité et la décence revient à asséner que l'intolérable sera désormais passible du jugement moral mais également légal (le second redoublant ici le premier). Cet article de la proposition de loi est perçu comme l'un des plus importants et d'aucun souligne la nécessité de l'association de ces trois termes malgré une sémantique contiguë. Il est bien consigné que « le respect, c'est le sentiment de réserve, de retenue, de déférence; la dignité, c'est le sentiment de gravité, de grandeur, et même de noblesse ; la décence enfin, c'est le sentiment de délicatesse, de discrétion, parfois même de modestie » (R. Yung lors du vote de l'article 9 de la proposition de loi $n^{\circ} 3186$, le 22 juin 2006). Le droit en convoquant le sentiment se fait sensible, sensible aux êtres, intimiste et modeste. Ce credo du respect est conforme à l'idée que dans une société qui valorise l'autonomie individuelle, où chaque individu est maître de son destin et de ses choix, la loi doit être un garant à même de protéger la personne humaine (surtout lorsque celle-ci n'est plus en vie). M. Hanus, président du Comité National d'Éthique du Funéraire, place ce motif moral au centre de la « ritualité » contemporaine à renforcer ou à créer. Il n'a de mots assez durs pour qualifier l'attitude qui consiste à procéder à la crémation sans autre forme de procès, dès l'arrivée du cercueil au crématorium ${ }^{22}$.

Il est toutefois légitime de se demander de manière pragmatique à quoi correspondent exactement la dignité, la décence et le respect d'un 
corps crématisé. Si ces valeurs parlent à tous dans l'absolu, le contenu des actions qu'elles diligentent est-il identique pour tout un chacun ? Dès lors, c'est davantage le non-respect, l'indécence et le manque de dignité qu'il faut saisir. D'où la prégnance de ces cas, cas atypiques, honteux, indicibles, inqualifiables. On le voit, le passage du constat qui fait état d'occurrences multiples à une qualification de ces occurrences comme déviantes est aisé mais par trop hâtif. Pourtant, la philosophie de cette loi repose sur une conception globale, laquelle exclut de se fonder sur le cas en tant qu'accident, comme le suppose la jurisprudence. C'est le caractère anomique des pratiques qui est mis en évidence, avec la nécessité absolue d'un garde fou - la loi étant la mieux à même de définir ce qui doit être. Par la voix du ministre délégué aux Collectivités territoriales, le texte discuté est présenté comme un gage aux bonnes mœurs visant à condamner les dérives actuelles, d'où son fondement éminemment moral : «ces situations sont choquantes, car elles soulignent ponctuellement une négligence, voire un désintérêt total pour le souvenir du défunt. Loin d'être passif, ce désintérêt se traduit par un abandon $d u$ souvenir d'une manière radicale, d'ailleurs totalement contraire à la décence due aux morts, pourtant très prégnante dans notre pays. Ces situations sont, à ce titre et fort heureusement, encore marginales, mais il appartient aux pouvoirs publics d'anticiper ce mouvement et de contrer ce qui pourrait être un comportement plus systématique » (discours introductif de B. Hortefeux, discussion en séance publique, le 22 juin 2006).

Le travail de la loi se fait vigilant, mais également caution. Au-delà du respect de la loi et des conventions, la volonté de décrire les bonnes mours et de les favoriser est clairement affichée. Le principe de « dignité de la personne humaine » doit ainsi s'étendre à la personne décédée quel que soit son choix en matière de sépulture. En interdisant l'usage (notamment commercial) des cendres et leur transformation, il est question de leur donner un statut et de punir toute instrumentalisation. Dans une société qui valorise le corps et la vie, promouvoir le respect des morts par le droit devenait une nécessité !

\section{Définir un modèle égalitaire des sépultures}

Le consensus repéré sur la nécessité de doter le cadavre et les cendres d'un statut s'étiole lorsqu'il s'agit d'envisager le devenir et plus spécifiquement la destination ultime de cellesci. La loi actuellement en vigueur est évasive et permet bien des possibilités. En la matière, le potentiel (voire le virtuel) et l'actuel tendent à s'amalgamer car aucune pratique ou presque n'est passible d'une sanction légale. Petit rappel, les cendres peuvent être : conservées à titre privé, divisées en plusieurs urnes ou amalgamées avec d'autres cendres; dispersées (tout ou partie) en tout lieu autre que la voie publique, immédiatement ou plusieurs années après le décès ; placées dans un caveau familial ou scellées sur celui-ci, déposées dans un columbarium ou un cavurne; dispersées dans un « jardin du souvenir »... En outre, même les principes du droit sont assouplis. Ainsi, P. Belhassen précise que suite à une demande de crématistes parisiens riverains de la Seine, il a été autorisé (certes à demi-mots) de procéder à la dispersion des cendres dans les eaux du fleuve, voie publique ${ }^{23}$. De même, si le droit proscrit des inhumations conjointes de corps 
dans un unique cercueil (à l'exception de deux enfants mort-nés ou de la mère et d'un enfant mort-né), il est possible d'inhumer un cadavre avec à ses côtés tout ou partie des cendres d'un proche décédé auparavant.

Ce qui est encore plus surprenant dans ce contexte, c'est que personne, pas même les sociologues et les opérateurs funéraires, n'est en mesure de dire ce qu'il advient des cendres à l'échelle d'une société telle que la France. Si les urnes sont remises à la famille dans $71 \%$ des $\operatorname{cas}^{24}$, seul chiffre tangible, cela ne veut aucunement dire qu'elles sont conservées par les proches à leur domicile. Il est donc impossible de connaître la destination réelle des cendres. Qui plus est, les restes d'une même personne peuvent avoir des trajectoires plurielles. Il n'est pas rare que l'urne rejoigne un buffet pour se loger à côté des photographies du défunt, pour quelques mois ou années plus tard être reléguée en des endroits moins visibles, puis être dans un troisième temps déposée dans un cimetière ou voir son contenu dispersé ${ }^{25}$. Il va de soi que la dispersion est sans alternative, à moins qu'elle ne soit réalisée sur un « échantillon ».

Face à un manque criant de données empiriques, il est intéressant de savoir quelle est la destination souhaitée pour les cendres chez ceux qui ont choisi la crémation. Telle est la question posée par la Chambre Syndicale Nationale de l'Art Funéraire en $2005^{26}$. Aucune destination n'est plébiscitée. Les réponses sont fort éclatées, preuve qu'il n'existe pas en la matière de modèles, encore moins de pratiques idéalisées et standardisées. Le peu de «médiatisations 》 des pratiques et l'absence de véritable(s) norme(s) laissent cours à un ensemble assez indéfini de comportements. En tout état de cause, le souhait d'une conservation durable au domicile de la famille est très minoritaire $(6 \%)^{27}$.

Cette absence de norme pourrait être perçue comme un contexte favorable à l'exercice du libre arbitre. La thèse d'une individualisation manifeste des pratiques qui voudrait que chaque individu se fasse sa « propre religion » et assume pleinement ses actes est à nuancer. Il serait en effet possible d'analyser cette situation comme l'émergence d'une multitude de normes, autant de repères que chacun pourrait se construire pour justifier le bien-fondé de ses choix. Or, cette compétition normative se constate indubitablement du point de vue du législateur, des promoteurs de la loi ou de ses détracteurs, elle est un enjeu crucial pour les associations crématistes, elle est également en discussion du côté des opérateurs funéraires ${ }^{28}$, de là à penser qu'elle a une quelconque prégnance sur le terrain des endeuillés serait aller trop vite en besogne. Le désarroi semble plus à même de qualifier les pratiques et les doutes relatifs au devenir du corps (le questionnement étant plus large que les cendres, puisque la première décision concerne le fait d'opter pour la crémation ou l'inhumation), qu'une affirmation d'un choix raisonné que l'on souhaiterait voir admis de tous. Les études récentes sur la crémation font état d'un trouble des proches à l'égard des cendres et de l'urne funéraire en tant qu'objet. Il semble subsister un véritable fossé entre une décision, mûrie ou non, idéologique ou pas, et le moment lui-même de l'acte de crémation ${ }^{29}$.

- L'entrée du cercueil dans le four crématoire, qui impose de façon irréversible que l'enveloppe 
corporelle ne sera plus à jamais ;

- La remise des cendres à la famille, parfois dans les heures qui suivent la cérémonie des funérailles (dorénavant, les professionnels préconisent un délai, certes court, entre l'incinération et la remise de l'urne cinéraire à la famille) ;

- La question épineuse de la destination des cendres qui, si elles sont dispersées trop rapidement évacue toute possibilité de trace et rendent tangible l'image d'un corps fantomatique (même si le geste a été vécu dans une certaine sérénité) et si elles sont conservées à titre privé deviennent rapidement encombrantes, car qu'en faire?

Toutes ces étapes sont ardues, non pas qu'elles fassent regretter l'acte crématiste, mais elles n'appartiennent pas à une culture constituée. En train de se faire, celle-ci est à l'épreuve du présent, des errements de chacun et oscillent entre une norme en formation, des bricolages, des espoirs intimes ${ }^{30}$. D'une certaine manière, le législateur prend acte de cette difficulté et souhaite étayer les pratiques en édifiant un système de valeur. Il définit par-là même une culture de la trace, voire de la traçabilité ${ }^{31}$. L'idée d'une indispensable médiation publique, d'un droit de regard qui énoncent le cadre des bonnes et mauvaises pratiques anime le législateur. Il s'agit de défendre la nécessité d'une destination « normale » des corps et des cendres, laquelle implique l'existence d'un sanctuaire public qui vise à séparer les vivants et les morts. En effet, comment imaginer dans un pays de tradition chrétienne, où le souvenir des morts est fort encadré et localisé, une telle ouverture des pratiques? Qui plus est, face à une absence réelle de référentiels communs, le législateur s'est saisi de cette apparente contradiction entre une tradition et des pratiques centrifuges sans apparents desseins.

Dans ce contexte, le législateur a souhaité en revenir à une vision traditionnelle du rapport aux défunts et promouvoir, en la publicisant, une vision du monde partagée. Tel est le point de vue affiché : "le respect dû à la mémoire de ceux qui nous ont précédés définit une civilisation. Il nous appartient, aujourd'hui, avec modestie, mais avec clarté, de faire ouvre de civilisation " (J.-P. Sueur, discussion en séance publique au Sénat, le 22 juin 2006). Ce qui est défendu ici c'est le caractère laïc des funérailles et plus avant l'égalité des modes de sépulture, avec en toile de fond une obligatoire sédentarisation. Pour le devenir des cendres, il paraît logique de proposer une destination qui soit conforme à la philosophie du cimetière républicain tel qu'il a été mis en place par les lois adoptées au début du $X^{\mathrm{e}}$ siècle : avec une inscription dans un espace-temps et la possibilité pour tous d'un recueillement en un lieu identifié. Cependant, cette position est controversée. L'étude du débat parlementaire est riche d'enseignements. Les promoteurs de la loi stipulent la nécessité de doter l'ensemble des pratiques funéraires d'un cadre laïc et républicain, y compris et surtout pour la crémation. Les valeurs qui fondent la Nation sont convoquées, mettant en balance la Liberté et l'Égalité. Le droit trouve ici matière à l'expression de son essence même, avec cette allégorie de l'équilibre juste. D'autres, comme B. Hortefeux, ministre délégué aux Collectivités territoriales, sont partagés entre la nécessité d'une loi qui énonce un cadre et la libre expression de chacun: "s'attacher à légiférer dans ce domaine exige d'avoir à l'esprit, à chaque mesure envisagée, l'équilibre 
nécessaire entre l'encadrement des pratiques funéraires, des opérations entourant le décès et le respect de chacun » (B. Hortefeux, discussion en séance publique au Sénat, le 22 juin 2006). Il met en balance les aspects techniques et juridiques et les aspects personnels, intimes, éminemment subjectifs. Dès lors, la décision de ne plus permettre la conservation des cendres à titre privé est dans cette perspective critiquée. La liberté fait débat. Le ministre insiste et prend part au débat en se faisant l'écho des échanges qu'il a pu avoir avec des particuliers, mais surtout avec des associations de libres-penseurs : " si je suis réellement attaché aux termes du projet de décret sur la destination des cendres, qui requiert - je ne cesse de le marteler - un équilibre entre le principe du respect de la volonté du défunt et l'implication de la sphère publique, la présente proposition de loi va plus loin dans la limitation de la liberté du futur défunt et pourrait, dès lors, susciter un vrai débat, en rendant impossible le dépôt d'une urne dans une propriété familiale. J'appelle donc votre attention sur les équilibres qu'elle pourrait modifier, même si je suis tout à fait prêt à reconnaître la logique du dispositif proposé privilégiant le dépôt dans une enceinte publique. S'il y a là, je le conçois, une vraie logique, nous ne pouvons, en revanche, faire l'économie d'un débat sur cette restriction de liberté » (B. Hortefeux, idem). Du point de vue du gouvernement, plus que le contenu, c'est la rapidité avec laquelle le législateur s'est saisi de cette question et comment il en est venu à verrouiller les marges de liberté qui est en cause. La démarche est perçue comme « un peu violente, un peu trop brutale», parce qu'elle "présente l'inconvénient de faire passer du rien au tout » (B. Hortefeux, idem).
Face à l'interdiction d'un usage privé des cendres, et donc de conserver l'urne à domicile, c'est à l'élaboration d'une autre liberté, que le rapporteur J.-R. Lecerf nous convie. Certes, l'on est face à la suppression d'une liberté, celle de l'appropriation privative des cendres, mais la loi instaure une nouvelle liberté, celle de pouvoir se recueillir devant les restes d'un être cher ou admiré. Le culte du tombeau est remodelé, mais préservé, authentifiant que l'ensemble des modes de sépulture doit s'organiser de manière analogue et susciter un souvenir en commun. En appeler au modèle du cimetière public, laïc et républicain, c'est considérer que les morts, s'ils entretiennent un nécessaire lien de filiation avec les vivants, celui-ci ne peut s'établir dans la sphère privée et familiale qu'en la présence de substituts : photographies, objets ${ }^{32}$. En aucun cas le corps du défunt ou ses cendres peuvent avoir un caractère privatif. Le rapport frontal entre vivants et morts relève dans cette conception de la sphère publique, la chair n'ayant pas de caractère privatif. En conséquence, la mise en présence des corps passe nécessairement par une médiation (la tombe, le columbarium, le caveau de famille). En outre, s'il n'est parfois pas possible d'approcher un être admiré durant sa vie, sa sépulture elle, doit être accessible à tous. La privatisation des cendres remettait en cause le culte des tombeaux en empêchant nombre de personnes " d'aller faire leur deuil devant les restes d'un défunt, ce qui est toujours possible dans un lieu public » (J.-P. Sueur, discussion en séance publique au Sénat, le 22 juin 2006).

Le caractère public défendu ici renvoie à l'égalité. Le principe égalitaire est promu au rang des valeurs inaliénables et emblématiques. Être tous 
égaux devant la mort, voici une aspiration que le législateur se doit de conserver, selon les promoteurs. Tout simplement parce que si les hommes sont égaux en droit durant leur vie, ceux-ci doivent l'être également par-delà leur mort ! D'où cette réponse de J.-P. Sueur : "Monsieur le ministre, vous avez parlé de liberté. Si nous sommes bien sûr tous favorables à la liberté, la question mérite réflexion, car elle doit s'exercer dans le cadre des lois de la République. En matière d'inhumation, personne ne considère comme une atteinte à la liberté l'obligation d'inhumer dans un cimetière public. Je ne vois pas pourquoi le même raisonnement de principe ne s'appliquerait pas aux urnes » (J.-P. Sueur, idem). Or, le constat est fait que les deux modes quasi-exclusifs de sépultures, à savoir l'inhumation et la crémation, sont en train de prendre des voies diamétralement opposées, ce que n'accepte pas le législateur. Récemment, l'agrément d'un site cinéraire privé 
souhaite un jour être crématisée est en soi un élément culturel marquant. Le caractère récent de la demande crématiste a pour conséquence que les représentations et pratiques qui lui sont afférentes ne sont pas fixées. D'une certaine façon les « excès » actuels attestent d'un travail d'élaboration, d'imagination et de mise en conformité. Malgré tout, la loi dit autre chose qu'un simple cadre. La dimension technique est essentielle mais non suffisante. En dernière instance, est-ce vraiment l'éclatement des pratiques qui est visé? N'est-ce pas plutôt la volonté des pouvoirs publics de normaliser, voire de moraliser, tant qu'il en est encore temps, les pratiques funéraires contemporaines qui sortent d'un cadre établi ?

Quand l'on étudie le contenu de cette loi, en particulier le débat qui a conduit à son vote (notamment sur la destination et le statut des cendres), on se rend compte qu'à défaut de modèles opératoires, c'est à une éthique de la mort que le public est invité, une morale raisonnable et pragmatique. La loi est finalement assez peu explicite, si toutefois elle est catégorique sur certains aspects, tel le respect, l'égalité, la localisation de la mort. Dès lors, il n'est pas sûr qu'elle sera en mesure de répondre aux prérogatives qui lui sont conférées au-delà de l'émergence d'un modèle. D'un point de vue sociologique, la question qui se pose est de savoir si l'éthique est ce qui préfigure l'émergence de pratiques? Ainsi, la morale peut-elle à ce point envahir le champ de l'action pour devenir seul référent ou en tout état de cause l'horizon ultime. «S'il n'existe pas de réponses toutes faites aux interrogations éthiques que soulèvent, à leurs différents temps, les pratiques de crémation, nous pouvons cependant tenter de les poser espérant ainsi faire progresser l'usage des pratiques recommandables $»^{36}$. Cette requête du Président du Comité National d'Éthique du funéraire nous dit là quelque chose qui redouble la loi. Tout est dit dans ce bel amalgame entre éthique et pragmatisme, qui met en forme une norme visant à énoncer les «bonnes» pratiques. Mais lesquelles? Cette réflexion n'est pas sans nous rappeler l'émergence de la «bonne mort» telle qu'elle a pu être configurée à propos des soins palliatifs notamment ${ }^{37}$. Entre recommandation et prescription la marge est infime. C'est l'idée également, qu'un peu vaut mieux que rien, donc que la néo-ritualité à laquelle nous sommes conviés est davantage supportable que des pratiques éparses, dont certaines prennent le risque de sortir des sentiers battus. Si la symbolisation fait défaut, c'est l'éthique qui prend le relais avec il faut bien le dire une instrumentalisation tant des pratiques que des valeurs avec un alignement sur ce qui existait déjà. Certains verront dans cette loi l'occasion ratée d'inventer une autre forme de ritualité funéraire, sans pour autant remettre en question la légitimité de légiférer sur un thème tel que celui-ci.

La nécessité d'en recourir à la loi, à une forme tangible qui fait trace et qui énonce le légitime, le normal et le juste, implique précisément que les régulations ne s'opèrent pas dans les pratiques quotidiennes et que les représentations contemporaines ne promeuvent pas un rapport aux morts satisfaisant. Si une loi dans le contexte actuel parait légitime, comment ne pas en percevoir les contreparties et surtout voir ce qu'elle énonce. Le parti pris a été jusqu'alors 
de repenser les pratiques actuelles à l'aune des pratiques ancestrales. Or, cette volonté de recourir à la crémation, puisque malgré tout ce mode de sépulture est un choix, fait émerger le désir d'un autre type de prise en charge. Lequel est peu pris en compte ici, puisque le propre de cette loi est d'uniformiser les pratiques en reposant sur une culture univoque du rapport à la mort, celle d'un souvenir localisé impliquant une traçabilité des corps et le dépôt de ceux-ci.
$\mathrm{N}$

$\mathrm{N}$

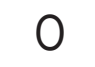

1. Voir V. Souffron, Brûler et non pourrir. Pour une socio-anthropologie de la crémation, Toulouse, Thèse de doctorat de sociologie, 1999.

2. Les prévisions de l'Association française d'information funéraire (Afif) établissent que les pratiques crématistes devraient s'établir à $35 \%$ en 2020, puis à 49\% en 2030. L'équilibre tant symbolique de la moitié des sépultures serait atteint dans moins d'un quart de siècle, changement tout à fait brutal à l'échelle des siècles passés.

3. P. Ariès, Essais sur l'histoire de la mort en Occident du moyen âge à nos jours, Paris, Seuil, 1975, et L'homme devant la mort, Paris, Seuil, 1977. M. Vovelle, Mourir autrefois, Paris, Gallimard, 1974 et La mort en occident de 1300 à nos jours, Paris, Gallimard, 1983 (réédition 2001). J.-D. Urbain, L'archipel des morts. Cimetières et mémoire en Occident, Paris, Petite bibliothèque Payot, 1998 (1ère édition 1989).

4. Le principe du choix des modes de sépultures est posé en 1887. La crémation devient possible mais n'est pas encore effective. Il faudra attendre 1889 pour que le règlement d'application soit promulgué, la première crémation ayant lieu en janvier de la même année sur un garçon de 11 ans. Voir P. Belhassen, La crémation : le cadavre et la loi, Paris, L.G.D.J., Panthéon-Assas, 1997, p. 61.

5. Voir J. Lalouette, «La libre pensée, l'Eglise et la crémation », Le mouvement social, n¹79, 1997, pp. 81-91.

6. Cette proposition de loi fait suite à une première proposition de loi de M. J.-P. Sueur relative à la législation funéraire, ${ }^{\circ} 464$, déposée le 7 juillet 2005 , laquelle a été renvoyée à la commission des lois constitutionnelles, de législation, du suffrage 
universel, du règlement et d'administration générale. La commission des lois saisie au fond a nommé J.R. Lecerf rapporteur le 7 juin 2006 (rapport n³86 déposé le 13 juin 2006). La discussion en séance publique a eu lieu le 22 juin 2006 (TA n¹11). La proposition de loi relative à la législation funéraire (n³186) adoptée par le Sénat a été déposée à l'Assemblée Nationale le même jour et renvoyée à la commission des lois constitutionnelles. Il était prévu dans un premier temps que la loi soit examinée avant le 1er novembre 2006, puis en mars 2007. Les échéances électorales (et la fin de la session parlementaire) ont repoussé son examen. Un décret n²007-328 du 12 mars 2007 publié au JO du 13 mars 2007 a pris acte de certaines modifications prévues par la proposition de loi, notamment l'expression « famille » est remplacée par «la personne ayant qualité pour pourvoir aux funérailles». En outre, la remise de l'urne à titre privé est possible si le défunt en a fait la demande de son vivant, pour cela la conservation au domicile ainsi que la dispersion en «pleine nature» doivent faire l'objet d'une déclaration auprès « du maire de la commune du lieu du dépôt $»$. Les lignes de partages observées dans le débat parlementaire sont confirmées. B. Hortefeux, ministre des Collectivités territoriales, indique dans un communiqué que ce décret a pour but de réformer le régime de la crémation et «s'assurer de l'existence d'un minimum de garanties en matière de destination des cendres ». Pour sa part, J.-P. Sueur ne se contente pas de cette première étape et indique clairement que ce décret ne peut «se substituer à la proposition de loi qui a été adoptée, à [son] initiative, par le Sénat unanime le 22 juin dernier qui traite d'un grand nombre de questions relatives à la législation funéraire, et qui relèvent clairement de la loi », communiqué du 13 mars 2007.

7. S. Nonnis Vigilante, «Encadrer la liberté : le statut et la destination des cendres dans le projet de réforme de la législation funéraire (2005-2006) », Quaderni, «Le Thanatopouvoir. Politique de la mort», ${ }^{\circ} 62$, Hiver 2006-2007, pp. 57-67.

8. Il est notamment le promoteur de la loi du 8 janvier 1993 qui a abrogé le monopole communal et a redéfini le secteur des pompes funèbres, ouvrant le marché à la concurrence. La loi a ainsi consacré la liberté pour les familles de choisir leur opérateur funéraire.

9. On peut imaginer que ce registre sera informatisé, seule trace publique du souvenir du défunt. La miniaturisation de la mort, définie par J.-D. Urbain, ou plus avant la logique de disparition défendue par $\mathrm{P}$. Baudry trouve là matière à réflexion. En effet, comment ne pas se questionner sur ce type de mise en mémoire qui correspond davantage à un stockage de données, qu'à un processus mémoriel! Voir J.-D. Urbain, L'archipel des morts, op. cit., p. 233 et P. Baudry, Violences invisibles. Corps, monde urbain, singularités, Bègles, Editions du passant, 2004.

10. P. Baudry, La place des morts. Enjeux et rites, Paris, Armand Colin, 1999.

11. H. Rabault, «Le problème de l'interprétation de la loi : la spécificité de l'herméneutique juridique », Le Portique, « La loi », n¹5, 2005.

12. P. Belhassen, La crémation : le cadavre et la loi, op. cit., p. 86.

13. J.-D. Urbain, La société de conservation. Sémiologie des cimetières en Occident, Paris, Payot, 1978. Il est d'ailleurs intéressant de constater que la formation des opérateurs funéraires, notamment des «maîtres de cérémonie », prend acte de cette idéologie en mentionnant par la voix du psychologue que le terme de cadavre ne doit jamais être employé lorsque l'on s'adresse à une famille touchée par un deuil.

14. Une société suisse Algordanza propose de 
convertir les cendres en diamant par une activité de raffinage. Voir www.algordanza.fr.

15. La société Space Services basée aux États-Unis offre la possibilité d'envoyer en orbite (dans une capsule) une petite partie des cendres provenant de la crémation d'un corps. Deux formules existent : 1 gramme de cendres en orbite vous coûtera $995 \$$, $5300 \$$ si vous optez pour 7 grammes. Le corbillard spatial sera assuré par une fusée Falcon I, laquelle pourra emporter deux cent défunts. Le vol est public, les familles pourront y assister et il est prévu d'organiser des cérémonies funèbres. Voir www. spaceservicesinc.com.

16. En son temps, L.-V. Thomas avait mentionné la plus grande circonspection à l'égard de certaines pratiques qui revenaient à se débarrasser à la va-vite des corps, produisant un amalgame entre le cadavre et les déchets. Voir L.-V. Thomas, Anthropologie de la mort, Paris, Payot, 1975 ; et Rites de morts. Pour la paix des vivants, Paris, Fayard, 1985.

17. Cour d'appel de Paris, arrêt du 27 mars 1998, in Codepratique des opérations funéraires, G. d'Abbadie et C. Bouriot, 3e édition, Paris, Le Moniteur, 2004.

18. Arrêt rendu à Douai le 7 juillet 1998, in Code pratique des opérations funéraires, G. d'Abbadie et C. Bouriot, op. cit.

19. L'inhumation dans un caveau repose sur de nombreuses prescriptions concernant le cercueil, la sépulture, le terme de la concession. Toute sépulture est protégée par le droit. La profanation des cimetières et des cadavres est lourdement sanctionnée. La violation de sépulture condamne les atteintes au respect dû aux morts et les dégradations des lieux de culte. Le code pénal stipule que «toute atteinte à l'intégrité du cadavre, par quelque moyen que ce soit, est punie d'un an d'emprisonnement et de $15000 €$ d'amende. La violation ou la profanation, par quelque moyen que ce soit, de tombeaux, de sépultures ou de monuments édifiés à la mémoire des morts est punie d'un an d'emprisonnement et de 15000 euros d'amende. La peine est portée à deux ans d'emprisonnement et à $30000 €$ d'amende lorsque les infractions définies à l'alinéa précédent ont été accompagnées d'atteinte à l'intégrité du cadavre », article 225-17 du Code pénal. Les peines sont encore plus lourdes si les motivations du geste sont assorties d'un caractère racial, ethnique ou religieux (article 225-18).

20. On note une professionnalisation de l'acte de crémation au crématorium, cet acte étant dévolu à des opérateurs funéraires. Les temporalités de l'action s'organisent comme suit: l'accueil de la famille et des proches, l'au revoir au mort avec une cérémonie civile au crématorium, la séparation qui, après un geste d'adieu, ordonne le départ du cercueil vers le four. C'est au rythme de musiques choisies pour l'occasion, de discours de l'officiant et de lecture de textes par la famille, que se déroule le cérémoniel qui est entrecoupé de silences et recueillements. « Tout » est explicité par le maître de cérémonie qui vise à rendre transparentes chacune des opérations. Le moment de l'introduction dans le four étant particulièrement éprouvant, il est conseillé aux endeuillés de se réunir dans des salons adjacents afin de parler du défunt, de boire un verre de l'amitié. Voir M. Hanus, "Le cadavre crématisé », Etudes sur la mort, $\mathrm{n}^{\circ} 129,2006$, p. 139.

21. Le code civil mentionne le respect dû au corps humain, celui-ci est inviolable et ses éléments et ses produits ne peuvent faire l'objet d'un traitement patrimonial (article 16 du Code civil). Toutes les juridictions (tribunal de grande instance, conseil d'État...) ont appliqué ces principes au cadavre avec la poursuite du respect de la personne humaine après la mort, mais ne pouvaient le faire concernant les cendres, puisque la loi permet leur dispersion, leur partage et leur amalgame. En outre, la possession 
à titre privé d'une urne cinéraire n'autorise aucun contrôle.

22. "Pratique scandaleusement inhumaine qu'il ne faut cesser de dénoncer jusqu'à ce tout le monde ait conscience de ce qu'il est: une horreur! Une honte! Car les rites sont nécessaires, ils ne sont pas en option », in «Le cadavre crématisé », art. cité, p. 137. M. Hanus est l'actuel président de la Société de Thanatologie, laquelle société avait été dirigée par L.-V. Thomas.

23. P. Belhassen, La crémation: le cadavre et la loi, op. cit., p. 35 ; et G. Rignault, La crémation, Thèse médecine, Tours, 1983.

24. Source émanant de la Chambre syndicale nationale de l'art funéraire, 2005, laquelle est conforme aux autres estimations.

25. Les opérateurs funéraires estiment qu'à l'échelle de plusieurs années, environ la moitié des urnes amenées au domicile sont ensuite déposées au cimetière dans un columbarium ou un caveau. Il n'est pas rare de retrouver des urnes vides abandonnées dans les allées d'un cimetière.

26. Souhaits exprimés quant à la destination des cendres: dispersion dans un milieu liquide $14 \%$, dispersion dans un jardin privé $13 \%$, ne sait pas $12 \%$, urne dans un caveau familial, $11 \%$, dispersion dans la nature $10 \%$, urne dans un monument funéraire $10 \%$, dispersion dans un jardin du souvenir $9 \%$, urne conservée chez la famille $6 \%$, urne dans un columbarium $5 \%$, autre $4 \%$, dispersion en montagne $4 \%$, d'autres décideront $3 \%$, urne dans un jardin $1 \%$. Source enquête : Chambre syndicale nationale de l'art funéraire, 2005.

27. L'écart entre $71 \%$ des urnes remises aux familles et $6 \%$ qui souhaitent une conservation à titre privé est éloquent. Cela peut rendre compte de deux situations tout à fait différentes mais néanmoins non contradictoires: soit les familles sont désarmées et ne savent pas ce qu'elles vont faire des cendres au moment du décès (la situation n'ayant pas été réfléchie en amont); soit la dispersion en pleine nature est majoritairement choisie car plus conforme aux valeurs dissipatives. Dans un cas le devenir des cendres est pensé, dans l'autre la détresse peut être réelle et indique une absence de « norme crématiste ». Les données complémentaires font cruellement défaut pour répondre à cette interrogation.

28. G. Clavandier, «La crémation : Des pratiques singulières à l'élaboration d'un cadre de référence », Etudes sur la mort. Thanatologie, «La crémation », $\mathrm{n}^{\circ} 132,2007$, pp. 65-86.

29. «La montée de la crémation: une nouvelle représentation de la mort », Consommation et modes de vie, Crédoc, n¹62, mars 2003 ; «La crémation, quels enjeux, quelles difficultés, quelles réponses?» Rapport du Crédoc réalisé pour le compte de la Chambre Syndicale Nationale d'Art Funéraire, 2002. Voir également, Le vécu et la perception du deuil et des obsèques, enquête qualitative réalisée auprès de familles endeuillées pour le compte du Comité Interfilière Funéraire, 1999.

30. C'est cet homme qui refusera une succession si son beau-père rejette l'idée de retirer les cendres de sa défunte de son garage aménagé en chapelle posthume. Ce sont ces enfants qui ont dit avec candeur à leur mère, que l'idée qu'elle se fasse incinérer et que ses cendres soient répandues dans les bois, était contraire à son goût immodéré des champignons : comment en manger sans dévorer maman ? C'est une amie, qui contrainte de déménager partira avec pour bagage, les cendres de son bébé mort-né. Autant de situations qui nous avons pu directement constater.

31. G. Clavandier, «Urne Funéraire. Des sépultures situées... des cendres évaporées », Actes du colloques international GDRI OpuS, La mort et le corps dans les arts d'aujourd'hui, l'Harmattan, à paraître 2008. 
32. Voir J.-H. Déchaux, Le souvenir des morts. Essai sur le lien de filiation, Paris, PUF, 1997.

33. Cette disposition est déjà en vigueur puisque le décret n²007-328 du 12 mars 2007 publié au JO du 13 mars 2007 l'a rendue obligatoire.

34. G. Clavandier, «La crémation : Des pratiques singulières à l'élaboration d'un cadre de référence », op. cit., pp. 70-73.

35. Voir C. Rivière, Les rites profanes, Paris, PUF, 1995.

36. M. Hanus, «Le cadavre crématisé », art. cité, p. 143.

37. Voir M. Castra, Bien mourir, Sociologie des soins palliatifs, Paris, PUF, 2003 ; et «Les soins palliatifs et l'euthanasie volontaire : l'affirmation de nouveaux modèles du "bien mourir" ", Actes du colloque Des vivants et des morts - «Des constructions de la bonne mort », Brest, pp. 113-120. Voir également les travaux de R.-W. Higgins, en particulier, « L'invention du mourant. Violence de la mort pacifiée », Esprit, n²91, janv. 2003, pp. 139-169. 